\title{
Systematic Reviews of Hospital Readmission Risk Screening: Reflective Case Study of Older Adults with Stroke
}

\author{
Jantra Keawpugdee ${ }^{1}$, Plernpit Boonyamalik ${ }^{2, *}$, Pimpan Silpasuwan², \\ Chukiat Viwatwongkasem ${ }^{3}$ and Ainat Koren ${ }^{4}$
}

\author{
${ }^{I}$ Ramathibodi School of Nursing, Faculty of Medicine, Ramathibodi Hospital, Mahidol University, \\ Bangkok 10400, Thailand \\ ${ }^{2}$ Department of Public Health Nursing, Faculty of Public Health, Mahidol University, \\ Bangkok 10400, Thailand \\ ${ }^{3}$ Department of Biostatistics, Faculty of Public Health, Mahidol University, Bangkok 10400, Thailand \\ ${ }^{4}$ Susan and Alan Solomont School of Nursing, Zuckerberg College of Health Sciences, \\ University of Massachusetts, Lowell, USA
}

('Corresponding author’s e-mail: plernpit.suw@mahidol.ac.th)

Received: 5 March 2021, Revised: 5 July 2021, Accepted: 15 July 2021

\begin{abstract}
Stroke remains a leading cause of death and disability, with older adults disproportionately affected. This study aimed to investigate stroke patients' short- and long-term readmissions to develop a new readmission risk screening tool (RRST) by conducting a systematic review and examining reflective cases reported to validate screening applications. Methods: A comprehensive search was conducted on 4 databases; eligible systematic reviews via CINAHL, MEDLINE/PubMed, Ovid UML, and Cochrane Library, with 14 research articles emerging to be content extracted as the $1^{\text {st }}$ draft. Expert opinion assessed findings, then revised them to develop a new RRST draft and checked it with a reflective quality check of 4 selected, screened cases. The review identified 14 studies using 5 screening tools. ISAR, TRST, and HOSPITAL score showed low to moderate validity and moderate to good reliability. The Risk Readmission Assessment Tool (RRAT) and LACE index validity and reliability scores were low to moderate. Hospital readmission predictors were hospital admission history, polypharmacy, cognitive and memory problems, the need for help, difficulties in walking, length of stay, and comorbid conditions. The 4 cases reported reflecting the developed RRST screening showed all common features. Value-added, the new RRST could accurately predict high-risk hospital readmission groups; the extended RRST tool screening quality is to be verified in clinical and community trials.
\end{abstract}

Keywords: Stroke, Hospital readmission, Systematic reviews, Case report, Screening tool

\section{Introduction}

Stroke is a significant cause of morbidity and mortality and the $2^{\text {nd }}$ leading cause of death worldwide in both developing and developed countries [1]. According to a rough estimate, its incidence has been increasing recently among Asian countries [2]. Stroke is a critical incident situation but probably results as a sign of long-term impacts on individual life. Hospital readmission of stroke patients has been one of the most fundamental problems of the healthcare system for the last decade $[3,4]$. The stroke readmission rate is an indicator of health care quality domains, including effectiveness, patientcenteredness, and efficiency [5]. The risk factors for stroke readmission are usually categorized as modifiable and nonmodifiable factors for risk prevention. However, little can be done to control nonmodifiable risk factors such as age and sex. It is essential to focus on managing the modifiable risk factors in this group of individuals to minimize risk [6]. However, there is no comprehensive, holistic measure or tool to screen readmissions for older adults with a stroke. Imperfections of existing screening tools result from: 1) Ineffective measurement, 2) No independent identification of factors, only correlation, 3) Too many items considered, 4) Association with other factors, and 5) Complexity of use.

Additionally, there is no quality readmission screening tool for older adults with stroke in primary practice in Thailand. There is a need to develop a quality screening tool for detecting hospital readmission risk among elderly stroke patients before discharge. Hence, this study aimed to identify and 
assess the stroke risk screening of elderly stroke patients before hospital discharge to home by exploring and synthesizing systematic reviews and testing the practicability and efficacy of a new screening tool. To effectively construct a risk screening tool for hospital readmission among elderly stroke patients is a development for addressing unplanned hospital readmission risk detection in a practical way for Thai stroke patients and provides significant added value to stroke care.

\section{Materials and methods}

\section{Study design}

A 2-step descriptive research design was conducted as follows;

Step 1: Systematic reviews in the medical and health science research to explore, select, sequencing, summarize, and evaluate RRST for older adults. Verifying content validity by indexes of Item-Objective Congruence (IOC) was performed by a panel of 9 experts and an in-depth interview of 2 selected experts directly involved in the comprehensive care and treatment of stroke patients to remove unclear or obscure items in the new tool construction.

Step 2: New tool implementation and evaluation in a pilot project in a stroke unit and 4 model case illustrations to predict the outcome measures. The total reflective cases were 30 stroke patients who were first admitted during 2018 - 2019 at Prasat Neurological Institute in Bangkok, Thailand. The subjects were reassessed through follow-up processes in the $1^{\text {st }}$ month $(2-4$ weeks $)$ and 6 weeks after hospital discharge.

\section{Measurement outcome}

For 1 primary outcome, the researchers conducted a review examining published research related to RRST for older adults. Four databases, CINAHL Plus with Full Text (Ebsco), MEDLINE/PubMed (Ebsco), Ovid UML Journals@Ovid (University of Massachusetts, Lowell Subscribed content), and Cochrane Library (Wiley), were searched using medical subject headings and keywords with the following terms: Readmission, Risk Assessment, Tools and Older adults. Terms were sought in titles, abstracts, or keywords. In addition to guarantee adequate and efficient coverage the relevant publications, Google Scholar was searched using similar medical subject headings and keywords.

The criteria for inclusion were: 1) The paper was in the English language, 2) It was original research, 3) The research was primarily of a quantitative type, 4) The paper was published during January 2000 - January 2017, 5) It included tools, qualities and factors related to hospital readmission of older adults, and 6) The study included outcome or results of readmission of older adults and the predictive validity of the tool on 1 or more outcomes. The exclusion criteria were: 1) It was either a systematic review, qualitative research, case study, or case report, and 2) It had a duplicate or irrelevant title or abstract. If the inclusion of the article was in doubt, a decision was made by 2 reviewers. The search flowchart and selection process are presented in Figures $\mathbf{1}$ and $\mathbf{2}$.

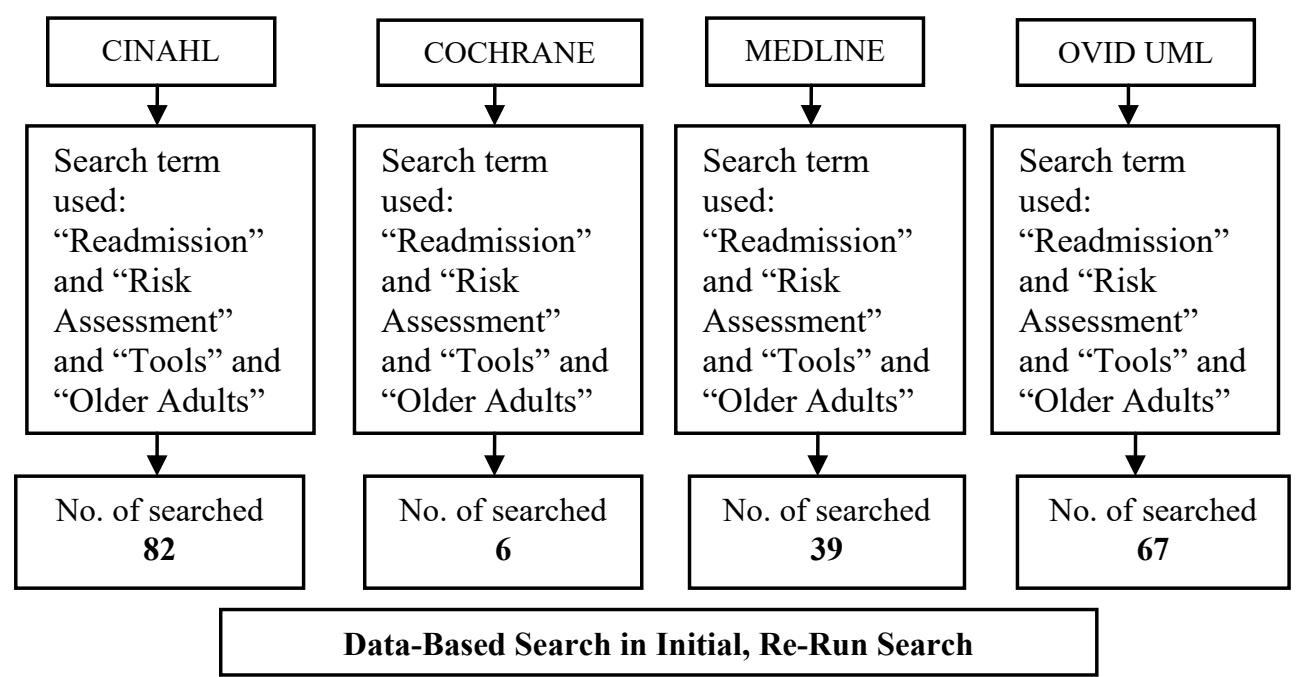

Figure 1 Numbers of research articles from 4 databases regarding Stroke Screening Tools. 


\section{Data extraction}

Data were extracted from the studies concerning the population, setting, sample size, reliability, validity, measurement, predictors, and outcome. We used the PRISMA checklist when writing our report [7]. Fourteen studies using 5 screening tools were identified. Based on the author's advanced scale screening tools, ISAR, TRST, HOSPITAL score, and the RRAT showed low to moderate validity and moderate to good reliability. The LACE index validity score was low to moderate. The most often identified predictors of hospital readmission were hospital admission history, polypharmacy, cognitive and memory problems, the need for help, difficulties in walking, length of stay, and comorbid conditions.

A pool of items emerged from systematic reviews and was developed for the initial prototype version of the $1^{\text {st }}$ draft of the Hospital-RRST.

194 Citations identified from electronic database searches

82 from CINAHL

39 from MEDLINE/PubMed

67 from Ovid UML

6 from Cochrane

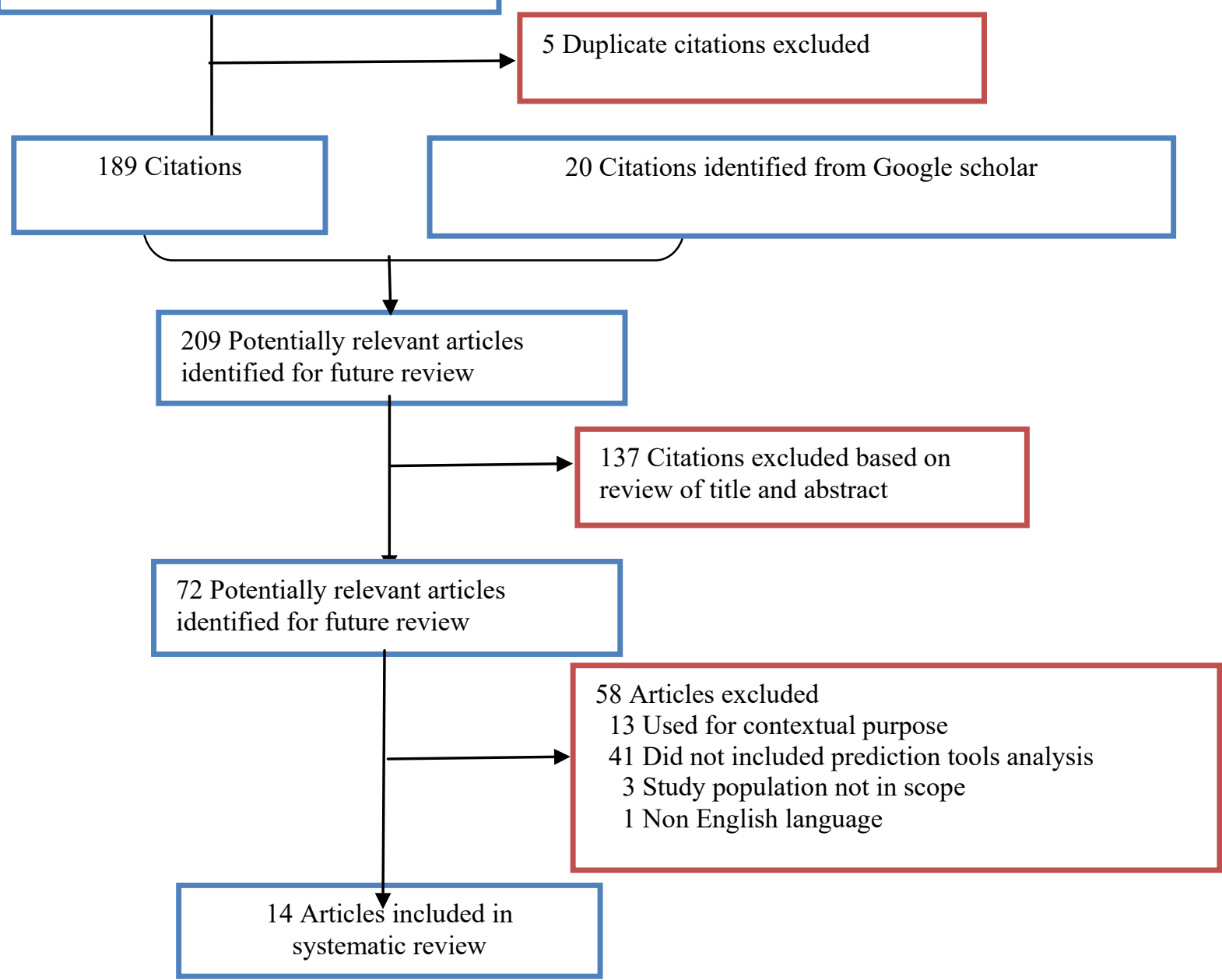

Figure 2 Steps of selecting, sequencing, and assessing the research articles from 4 databases regarding the Stroke Screening Tool. 


\section{Itemized reviews}

Nine experts gathered a panel for validating content validity. This study's panel included 4 medical doctors, 3 nurse instructors, and 2 head nurses studying and working in stroke care for older adults who assessed multiple factors affecting the hospital readmission risk screening of stroke in the elderly. After reviewing and revising the $1^{\text {st }}$ draft of RRST, the $2^{\text {nd }}$ draft of the RRST was constructed. Then a pilot study was conducted among 30 older adults with stroke during October 2019 - February 2020.

For research with human subjects, ethical considerations were necessary. Before implementation of the study protocol, approval was obtained by the Human Research Ethics Committee COA, No. MUPH, 2016-119 (Renewal until 2019). Furthermore, the Prasat Neurological Institute's human rights committee approved the study protocol number 049/2561.

\section{Data analysis}

Descriptive statistics applied indexes of IOC as the content validity Index; the proportion of items with expert's agreement should not be less than 0.8 [8].

\section{Results and discussion}

\section{Characteristics of studies selected}

Firstly, electronic database searches and reference lists yielded 209 citations. Through the elimination process depicted in Figure 2, 14 studies were included in this systematic review (Figure 1). Finally, 5 primary readmission prediction tools were identified: Identification of Seniors at Risk screening tool (ISAR); Triage Risk Stratification Tool (TRST), HOSPITAL score; RRAT; some of the studies used a combination of 2 tools, the ISAR and TRST tools [9-11], and the LACE index.

A standard outcome measure was the 30-day hospital readmission [12-15]. Several studies conducted repeated readmission measures at 30, 90, 120, and 365 days after discharge [9-11,16-19]. Most of the research studies did not specify the medical condition of older adults. The most commonly used study design was the prospective cohort study [12-15]. One study used an interventional evidence-based project with pre-and post-analysis [20].

\section{Description summary of the RRST}

The $1^{\text {st }}$ draft included 5 tools (62 items) described below:

\section{Identification of the Seniors-Risk Screening Tool (ISAR)}

The ISAR is a quick and easy screening tool first validated in an emergency department (ED) in Canada. The ISAR has 6 items that assess a person's needs for help regularly (i.e., home care, home nursing services, or support from relatives or others). It is related to the need for support (personal care) that is more than usual for self-care after illness, history of hospital admissions, visual problems, memory problems, and polypharmacy (more than 3 drugs). Most studies used the ISAR to predict older adult readmission to the ED. Some studies, such as Edmans et al. [21], added mental well-being and quality-oflife components to the assessment tool.

\section{Triage Risk Stratification Tool (TRST)}

The TRST was developed and primarily used to screen older adults in the ED in Switzerland, Belgium, Canada, and the United States of America. The TRST comprises 5 items: Cognitive impairment, difficulties of walking or transfer, polypharmacy, admission to the ED during the past month or hospitalization within the past 3 months [9-11,18]. Some studies added variables such as living alone with no caregiver. The reliability testing results showed the area under the curve was 0.61 with a cut-off score of 2 [18] and a sensitivity of $73 \%$, a specificity of $75 \%$, and a negative predictive value of $92 \%$. $[10]$.

\section{HOSPITAL score}

The studies included in this review used this tool to predict hospital readmission and internally validate a prediction model for potentially avoidable 30-day hospital readmissions [12,14]. The HOSPITAL score consists of 6 items: Low hemoglobin at discharge, discharge from an oncology service, low sodium level at release, procedures during a hospital stay, index admission type (urgent or emergency), and several hospital admissions during the previous year with the length of stay $\geq 5$ days. The validity test showed a $\mathrm{C}$ statistic of 0.72 , indicating good discrimination for hospital readmission [12]. 


\section{Risk Readmission Assessment Tool (RRAT)}

This tool's purpose was to determine whether the patient was at high risk for readmission $[13,15]$. The RRAT contains 7 items including: insurance status, marital status, having a regular physician, number of admissions within the past calendar year, length of stay, the comorbidity index score, and the physical component of Short Form-12 Health Survey. In some studies, such as Boraiah et al. [15], validity results were low to moderate. The reliability testing results showed the area under the curve was 0.61 with a cut-off score of 2 [18].

\section{LACE index}

This index aimed to quantify unplanned readmissions [20, 22]. The LACE index consists of 4 items: Length of stay, acuity of admission, comorbid conditions, and whether the ED was used in the 6 months before admission. The validity test showed that scores using the LACE index ranged from 0 to 19 and was very accurate at predicting outcome risk [22].

\section{The $2^{\text {nd }}$ draft of RRST}

Sixty-two items consisted of a stroke patient screening checklist modified to be a questionnaire comprised of 5 parts:

1) ADL (applied the original source from the Barthel Index) consists of 10 items $=100$ scored, rating scale; $10=$ able to do all, $5=$ do some, $0=$ cannot do. Additionally, the interpretation; 0 - 20 scored $=$ high risk, 25 - 90 scored $=$ middle risk, 95 - 100 scored $=$ low risk.

2) IADL (modified originality source from Instrument Activities of Daily Living Scale) consists of 8 items $=8$ scored (full scored), rating scale; $8=$ able to do, $2-7=$ do some, $0-1=$ cannot do. Additionally, the interpretation; 0 - 1 scored $=$ high risk, $2-7$ scored $=$ middle risk, 8 scored $=$ low risk.

3) Health Risk Behavior (HRB): (applied originality source from chronic care and expert opinion) all items were rated a 2-points; $2=$ able to do, $1=$ cannot do $(28$ items, full scored $=56 ; 100 \%)$. The summative response scale with HRB interpretation; $<20 \%=$ high risk, $\geq 20 \pm 79 \%=$ moderate risk, $\geq$ $80=$ low risk

4) ISAR (applied originality source from ISAR) consists of 7 items $=$ full scored $=14: 100$.

5) TRST (applied originality source from TRST) consists of 9 items $=$ full scored $=18: 100$.

On the ISAR and TRST, all items were rated on a 2-point summative response scale scored by rating, and the interpretation used the same values scored scaling of HRB. The RRST was calculated and improved in the $2^{\text {nd }}$ draft of the RRST.

\section{Validity} RRST.

The IOC was used to evaluate the $\operatorname{RRST}=0.936$, mean $=49$, and $\mathrm{SD}=13.360$ as a $2^{\text {nd }}$ draft of

\section{Reliability}

Cronbach's alpha coefficient examined the internal consistency reliability. The Cronbach's coefficient alpha was computed for the total scales and each sub-scale of the new tool. An acceptable coefficient was 0.70 or greater [23]. In general, correlation ranging from 0.30 to 0.50 ( -0.30 to -0.50$)$ is considered low positive/negative; correlation ranging from 0.50 to $0.70(-0.50$ to -0.70$)$ is moderate positive/negative; and correlation ranging from 0.70 to $0.90(-0.70$ to -0.90$)$ considered high positive/negative. A correlation above 0.90 is considered very high [24]. The RRST scored had coefficient correlation $=0.72$; it indicated the RRST scored measure had a slightly high level of tool reliability in specific time and space [25].

To develop the RRST, the researchers examined 4 patients' records and individual reports using the RRST screening score classified into 8 categories for contrast and comparison of each case's features, including clinical characteristics before discharge home, as shown in Table 1. 
Table 1 The 4 selected cases of elderly stroke patients using RRST before discharge to home classified by each crucial clinical aspect of the case reported individually.

\section{Patient Information}

\begin{tabular}{|c|c|c|c|c|c|c|c|}
\hline Case/characteristics & Age & Sex & status & Education & $\begin{array}{c}\text { Income } \\
\text { US\$/ year }\end{array}$ & Occupation & Family \\
\hline Case no. 1 & 61 & male & single & $\begin{array}{c}\text { Secondary } \\
\text { school }\end{array}$ & $\mathrm{n} / \mathrm{a}$ & monk & $\mathrm{n} / \mathrm{a}$ \\
\hline Case no. 2 & 80 & female & married & $\begin{array}{c}\text { Primary } \\
\text { school }\end{array}$ & 2,883 & Unemployed & $\begin{array}{c}\text { Living with } \\
\text { husband/wife } \\
\text { grandchildren and } \\
\text { relatives }\end{array}$ \\
\hline Case no. 3 & 80 & female & married & $\begin{array}{c}\text { Secondary } \\
\text { school }\end{array}$ & 2,883 & Unemployed & $\begin{array}{c}\text { Living with } \\
\text { husband/wife and } \\
\text { children }\end{array}$ \\
\hline Case no. 4 & 75 & female & married & $\begin{array}{c}\text { Primary } \\
\text { school }\end{array}$ & 2,523 & Unemployed & $\begin{array}{c}\text { Living with } \\
\text { husband/wife and } \\
\text { children }\end{array}$ \\
\hline
\end{tabular}

\section{Clinical Presentation}

\begin{tabular}{cccc}
\hline Case/characteristics & Diagnosis & Duration of Illness & Chief Complaint \\
\hline Case no. 1 & Ischemic Stroke & $17^{*}$ days & Weak left hand and leg 2.07 h. PTA \\
Case no. 2 & Ischemic Stroke & $14^{*}$ days & Ataxia 2 days PTA (before admitted) \\
Case no. 3 & Ischemic Stroke & $8^{*}$ days & Weak right hand and leg, dysarthria 2 days PTA \\
Case no. 4 & Ischemic Stroke & $7^{*}$ days & Weak left hand and leg, dysarthria 2 days PTA \\
\hline
\end{tabular}

\section{Diagnostic assessment}

\begin{tabular}{|c|c|c|c|}
\hline Case/characteristics & Sign/symptoms & Past History & NIHSS Scored \\
\hline Case no. 1 & $\begin{array}{l}\text { 2.07 h. PTA while eating, then Buddhist monk } \\
\text { felt weak left side (left hemiparesis), facial } \\
\text { weakness (minor left facial weakness), severe } \\
\text { dysarthria, alert and good consciousness, no } \\
\text { numbness. }\end{array}$ & $\begin{array}{l}\text { Diabetes, Hypertension, } \\
\text { Ischemic-Heart Disease status; } \\
\text { post coronary-artery-bypass } \\
\text { surgery (CABG), Chronic- } \\
\text { kidney disease (CKD). }\end{array}$ & $11^{*}$ \\
\hline Case no. 2 & $\begin{array}{l}\text { Two days PTA feeling vertigo, ataxia but can } \\
\text { stand, no weakness, good verbal ability, no } \\
\text { facial palsy. Today, at noon felt dysarthria, } \\
\text { ataxia, cannot stand, blurred vision in both } \\
\text { eyes. }\end{array}$ & $\begin{array}{l}\text { unknown underlying, social } \\
\text { alcohol drinking, no smoking }\end{array}$ & $4 *$ \\
\hline Case no. 3 & $\begin{array}{c}\text { Two days PTA, weak right hand and leg, } \\
\text { dysarthria, alert and good consciousness, no } \\
\text { numbness. }\end{array}$ & $\begin{array}{c}\text { Diabetes, Hypertension, and } \\
\text { chronic kidney disease (CKD). }\end{array}$ & $11 *$ \\
\hline Case no. 4 & $\begin{array}{l}\text { Two days PTA (last seen } 9 \text { pm - March 15, } \\
\text { 2019, had a normal sleep) relatively weak left } \\
\text { hand and leg (left hemiparesis), dysarthria, left } \\
\text { facial palsy, alert, less content of } \\
\text { consciousness, and followed } 1 \text { step command. }\end{array}$ & $\begin{array}{c}\text { Type } 2 \text { Diabetes, Hypertension, } \\
\text { and Dyslipidemia. }\end{array}$ & $14^{*}$ \\
\hline
\end{tabular}

\section{Diagnostic examination}

\begin{tabular}{cccc}
\hline Case/characteristics & MRI & Computed Tomography Angiography & Echo \\
\hline Case no. 1 & $\begin{array}{c}\text { Restrictive } \\
\text { diffusion at right } \\
\text { pons. }\end{array}$ & $\begin{array}{c}\text { Old hypo-dense lesion at right frontal lobe, } \\
\text { and hyper-dense lesion at pons and stenosis } \\
\text { of the M1 part of the right MCA basilar }\end{array}$ & $\begin{array}{c}\text { Severely impair LV systolic function, } \\
\text { EF 25 no intracardiac thrombosis. }\end{array}$
\end{tabular}
aneurysm. 
Case no. 2 No Hypo-density at right pons, remote lacunar infraction at the left lentiform nucleus, and posterior limb of the bilateral internal capsule.

Case no. 3 No Case no. 4 No
Hypo-density lesion at left pons.

Hypo-dense lesion of the right subcortical white matter and right periventricular, old hypo-density at the right occipital.
No

No

Good EF, good LV and RV systolic function, no intracardiac clot.

\section{Clinical Characteristics}

\begin{tabular}{|c|c|c|c|c|c|c|}
\hline Case/characteristics & \multicolumn{4}{|c|}{ Vital Signs } & \multicolumn{2}{|c|}{ Body Mass index (BMI) kg/m $\mathrm{m}^{2}$} \\
\hline Case no. 1 & \multicolumn{4}{|c|}{$\begin{array}{c}\text { Temp }=37^{\circ} \mathrm{C}, \text { Pulse }=100 / \mathrm{min}, \mathrm{RR}=22 / \mathrm{min}, \mathrm{BP}=142 / 80^{*} \\
\mathrm{mmHg}(\text { standard } \mathrm{BP} \leq 130 / 80 \mathrm{mmHg}) \mathrm{BW}=72 \mathrm{~kg}, \mathrm{HT}= \\
170 / \mathrm{cm}^{2}\end{array}$} & \multicolumn{2}{|c|}{$24.9^{*}$} \\
\hline Case no. 2 & \multicolumn{4}{|c|}{$\begin{array}{c}\text { Temp }=36.6^{\circ} \mathrm{C}, \text { Pulse }=64 / \mathrm{min}, \mathrm{RR}=12 / \mathrm{min}, \mathrm{BP}=149 / 70^{*} \\
\mathrm{mmHg}(\text { standard } \mathrm{BP} \leq 130 / 80 \mathrm{mmHg}) \mathrm{BW}=57 \mathrm{~kg}, \mathrm{HT}= \\
164 / \mathrm{cm}^{2}\end{array}$} & \multicolumn{2}{|c|}{21.3} \\
\hline Case no. 3 & \multicolumn{4}{|c|}{$\begin{array}{c}\text { Temp }=37.7^{\circ} \mathrm{C}, \text { Pulse }=100 / \mathrm{min}, \mathrm{RR}=20 / \mathrm{min}, \mathrm{BP}=170 / 80^{*} \\
\mathrm{mmHg}(\text { standard } \mathrm{BP} \leq 130 / 80 \mathrm{mmHg}) \mathrm{BW}=65 \mathrm{~kg}, \mathrm{HT}= \\
164 / \mathrm{cm}^{2}\end{array}$} & \multicolumn{2}{|c|}{$24.2 *$} \\
\hline Case no. 4 & \multicolumn{4}{|c|}{$\begin{array}{c}\text { Temp }=37^{\circ} \mathrm{C}, \text { Pulse }=84 / \mathrm{min}, \mathrm{RR}=22 / \mathrm{min}, \mathrm{BP}=151 / 86^{*} \\
\mathrm{mmHg}(\text { standard } \mathrm{BP} \leq 130 / 80 \mathrm{mmHg}) \mathrm{BW}=74 \mathrm{~kg}, \mathrm{HT}= \\
162 / \mathrm{cm}^{2}\end{array}$} & \multicolumn{2}{|c|}{$28.2 *$} \\
\hline \multicolumn{7}{|c|}{ 6. Laboratory Results } \\
\hline Case/characteristics & $\begin{array}{l}\text { Total } \\
\text { Cholesterol: } \\
\text { TC }\end{array}$ & $\begin{array}{l}\text { Fasting } \\
\text { Blood } \\
\text { Sugar: } \\
\text { FBS }\end{array}$ & $\begin{array}{l}\text { Renal } \\
\text { Function } \\
\text { Test: RFT }\end{array}$ & $\begin{array}{c}\text { Liver Function } \\
\text { Test: LFT }\end{array}$ & $\begin{array}{l}\text { Complete } \\
\text { Blood Count: } \\
\text { CBC }\end{array}$ & $\begin{array}{l}\text { International } \\
\text { Normalized Ratio: } \\
\text { INR }\end{array}$ \\
\hline Case no. 1 & $\begin{array}{c}\mathrm{HDL}=55 \\
\mathrm{mg} / \mathrm{dl}, \mathrm{LDL} \\
=106 \mathrm{mg} / \mathrm{dl}, \\
\text { Cholesterol } \\
=189 \mathrm{mg} / \mathrm{dl}, \\
\text { Triglyceride } \\
\mathrm{s}=130 \\
\mathrm{mg} / \mathrm{dl}\end{array}$ & $\begin{array}{c}\mathrm{FBS}= \\
177 \\
\mathrm{mg} / \mathrm{dl} \\
\mathrm{HbA} 1 \mathrm{C} \\
=7.1 \%\end{array}$ & $\begin{array}{l}\mathrm{BUN}=55.8 \\
\mathrm{mg} / \mathrm{dl}, \\
\text { Creatinine }= \\
3.34 \mathrm{mg} / \mathrm{dl}\end{array}$ & $\begin{array}{c}\text { AST }(\mathrm{SGOT})=20 \\
\mathrm{U} / \mathrm{L}, \mathrm{ALT}(\mathrm{SGPT})= \\
22 \mathrm{U} / \mathrm{L}, \mathrm{ALP}(\mathrm{Alk} . \\
\text { Phosphatase })=78 \\
\mathrm{U} / \mathrm{L}, \text { Total protein }= \\
7.5 \mathrm{~g} / \mathrm{dl}, \text { Albumin }= \\
3.4 \mathrm{~g} / \mathrm{dl}, \text { Globulin }= \\
4.1 \mathrm{~g} / \mathrm{dl}, \text { Total } \\
\text { bilirubin }=0.56 \\
\mathrm{mg} \%, \text { Direct } \\
\text { bilirubin }=0.25 \\
\mathrm{mg} \%\end{array}$ & $\begin{array}{c}\text { Hematocrit } \\
(\mathrm{Hct})=41.8 \%, \\
\text { Hemoglobin } \\
(\mathrm{Hb})=13.7 \mathrm{~g} / \mathrm{dl}, \\
\text { Platelet count }= \\
280,000 / \mathrm{L}\end{array}$ & $\begin{array}{c}\text { Prothrombin Time } \\
(\mathrm{PT})=12.4 \mathrm{~s}, \\
\text { Activated Partial } \\
\text { Thromboplastin } \\
\text { Time }(\mathrm{aPPT})=26.1 \\
\mathrm{~s} \text {, International } \\
\text { Normalized Ratio } \\
(\text { INR })=1.02\end{array}$ \\
\hline Case no. 2 & $\begin{array}{c}\mathrm{HDL}=63 \\
\mathrm{mg} / \mathrm{dl}, \mathrm{LDL} \\
=127 \mathrm{mg} / \mathrm{dl}, \\
\text { Cholesterol } \\
=211 \mathrm{mg} / \mathrm{dl}, \\
\text { Triglyceride } \\
\mathrm{s}=96 \mathrm{mg} / \mathrm{dl}\end{array}$ & $\begin{array}{c}\mathrm{FBS}= \\
102 \\
\mathrm{mg} / \mathrm{dl}, \\
\mathrm{HbA1C} \\
=5.03 \%\end{array}$ & $\begin{array}{c}\mathrm{BUN}= \\
15.11 \mathrm{mg} / \mathrm{dl} \text {, } \\
\text { Creatinine = } \\
0.97 \mathrm{mg} / \mathrm{dl}\end{array}$ & $\begin{array}{c}\text { AST }(\mathrm{SGOT})=32 \\
\mathrm{U} / \mathrm{L}, \mathrm{ALT}(\mathrm{SGPT})= \\
26 \mathrm{U} / \mathrm{L}, \mathrm{ALP}(\mathrm{Alk} . \\
\text { Phosphatase })=124 \\
\mathrm{U} / \mathrm{L}, \text { Total protein }= \\
6.5 \mathrm{~g} / \mathrm{dl}, \text { Albumin }= \\
3.3 \mathrm{~g} / \mathrm{dl}, \text { Globulin }= \\
3.2 \mathrm{~g} / \mathrm{dl}, \text { Total } \\
\text { bilirubin }=0.49 \\
\mathrm{mg} \%, \text { Direct } \\
\text { bilirubin }=0.12 \\
\mathrm{mg} \%\end{array}$ & $\begin{array}{c}\text { Hematocrit } \\
(\mathrm{Hct})=37.0 \%, \\
\text { Hemoglobin } \\
(\mathrm{Hb})=11.8 \mathrm{~g} / \mathrm{dl}, \\
\text { Platelet count }= \\
322,000 / \mathrm{L}\end{array}$ & $\begin{array}{c}\text { Prothrombin Time } \\
(\mathrm{PT})=12.0 \mathrm{~s}, \\
\text { Activated Partial } \\
\text { Thromboplastin } \\
\text { Time }(\mathrm{aPPT})=24.7 \\
\mathrm{~s} \text {, International } \\
\text { Normalized Ratio } \\
(\text { INR })=0.98\end{array}$ \\
\hline Case no. 3 & $\begin{array}{c}\mathrm{HDL}=50 \\
\mathrm{mg} / \mathrm{dl}, \mathrm{LDL}\end{array}$ & $\begin{array}{c}\mathrm{FBS}= \\
185\end{array}$ & $\begin{array}{c}\mathrm{BUN}= \\
27.03 \mathrm{mg} / \mathrm{dl},\end{array}$ & $\begin{array}{c}\text { AST }(\text { SGOT })=29 \\
\mathrm{U} / \mathrm{L}, \mathrm{ALT}(\mathrm{SGPT})=\end{array}$ & $\begin{array}{c}\text { Hematocrit } \\
(\mathrm{Hct})=34.7 \%,\end{array}$ & $\begin{array}{l}\text { Prothrombin Time } \\
(\mathrm{PT})=12.9 \mathrm{~s},\end{array}$ \\
\hline
\end{tabular}




$\begin{array}{lll}=46 \mathrm{mg} / \mathrm{dl}, & \mathrm{mg} / \mathrm{dl}, & \text { Creatinine }= \\ \text { Cholesterol } & \mathrm{HbA} 1 \mathrm{C} & 1.94 \mathrm{mg} / \mathrm{dl} \\ =109 \mathrm{mg} / \mathrm{dl}, & =6.6 \% & \\ \text { Triglyceride } & & \\ \mathrm{s}=64 \mathrm{mg} / \mathrm{dl} & & \end{array}$

Case no. 4

$\mathrm{BUN}=5$

$\begin{array}{cc}\mathrm{HDL}=33 & \mathrm{FBS}= \\ \mathrm{mg} / \mathrm{dl}, \mathrm{LDL} & 118 \\ =66 \mathrm{mg} / \mathrm{dl}, & \mathrm{mg} / \mathrm{dl}, \\ \text { Cholesterol } & \mathrm{HbA} 1 \mathrm{C} \\ =121 \mathrm{mg} / \mathrm{dl}, & =7.2 \% \\ \text { Triglyceride } & \\ \mathrm{s}=140 & \\ \mathrm{mg} / \mathrm{dl} & \end{array}$

18 U/L, ALP (Alk. Phosphatase $)=49$

$\mathrm{U} / \mathrm{L}$, Total protein $=$ $7.6 \mathrm{~g} / \mathrm{dl}$, Albumin = $2.9 \mathrm{~g} / \mathrm{dl}$, Globulin = $4.7 \mathrm{~g} / \mathrm{dl}$, Total bilirubin $=0.64$ $\mathrm{mg} \%$, Direct bilirubin $=0.33$ $\mathrm{mg} \%$ $\mathrm{mg} / \mathrm{dl}$, Creatinine $=$ $0.67 \mathrm{mg} / \mathrm{dl}$

$\operatorname{AST}(\mathrm{SGOT})=18$ $\mathrm{U} / \mathrm{L}, \operatorname{ALT}(\mathrm{SGPT})=$ 12 U/L, ALP (Alk. Phosphatase $)=37$ $\mathrm{U} / \mathrm{L}$, Total protein $=$ $6.5 \mathrm{~g} / \mathrm{dl}$, Albumin = $3.2 \mathrm{~g} / \mathrm{dl}$, Globulin $=$ $3.3 \mathrm{~g} / \mathrm{dl}$, Total bilirubin $=0.13$ mg\%, Direct

bilirubin $=0.22$ $\mathrm{mg} \%$
Hemoglobin $(\mathrm{Hb})=11.2 \mathrm{~g} / \mathrm{dl}$, Platelet count $=$ 222,000/L

Hematocrit $(\mathrm{Hct})=34.7 \%$, Hemoglobin $(\mathrm{Hb})=11.7 \mathrm{~g} / \mathrm{dl}$, Platelet count $=$ 255,000/L
Activated Partial Thromboplastin Time $($ aPPT $)=24.0$ $\mathrm{s}$, International Normalized Ratio $(\mathrm{INR})=1.06$

Prothrombin Time $(\mathrm{PT})=13.4 \mathrm{~s}$, Activated Partial Thromboplastin Time $(\mathrm{aPPT})=26.8$ $\mathrm{s}$, International Normalized Ratio $(\mathrm{INR})=1.1$

\section{Treatment}

\begin{tabular}{ccc}
\hline Case/characteristics & Drug & Drug Type \\
\hline Case no. 1 & Aspirin (325 mg) oral OD, Aspirin (81 mg) oral OD, and & Dual antiplatelet drug \\
Case no. 2 & Clopidogrel (75 mg) oral OD. & Antiplatelet drug \\
Case no. 3 & Aspirin $(325 \mathrm{mg})$ oral OD. & Antiplatelet drug \\
Case no. 4 & Clopidogrel $(75 \mathrm{mg})$ oral OD and Enoxaparin 0.6 cc SC OD & Antiplatelet drug \\
\hline
\end{tabular}

Table 2 The risk screening before discharge to home classified by each crucial clinical aspect of the cases reported individually.

\begin{tabular}{ccccccc}
\hline \multicolumn{7}{c}{ Risk Screening before Discharge Home } \\
\hline Case/characteristics & ADL & IADL & HRB & TRST & ISAR & \multirow{2}{*}{ Total score } \\
\hline Scored & Scored & Scored & Scored & Scored & 31 \\
Case no. 2 & $10^{*}$ & $1^{*}$ & 13 & $2^{*}$ & $5^{*}$ & 39 \\
Case no. 3 & $20^{*}$ & $1^{*}$ & 13 & $1^{*}$ & $4^{*}$ & 33 \\
Case no. 4 & $15^{*}$ & $1^{*}$ & 10 & $2^{*}$ & $5^{*}$ & 29 \\
\hline
\end{tabular}

Note: $*=$ High-risk score. The value is lower or higher than the reference value/Reference; ISO 15189 from Bureau of Laboratory 28 Quality Standards, Department of medical science, Ministry of Public Health.

Concerning risk screening before discharge to home, the ADL assesses problems with daily living, walking, movement, and eating. Among those, Case no.1 had a problem with defecation and urinary elimination. Regarding BMI $\mathrm{kg} / \mathrm{m}^{2}$, all 4 cases were overweigh (above standard) and had high Blood Pressure (except Case no. 3). 
According to the laboratory examination, all cases (except Case no. 2) had a high level of HbA1C (above the standard). Some of them had high BUN and Creatinine (above the standard). For CBC, they had a lower Hematocrit and Hemoglobin level, also Albumin. Cases no. 1, 3 and 4 had Diabetes Mellitus, Hypertension, and CKD as underlying diseases. The IADL factor was insufficient in all instances with HRB, ISAR, and HRST rated high risk combined with a long stay of hospital utilization.

\section{Discussion}

This study reviewed 14 research studies to summarize the validity, reliability, and predictive ability of 5 RRST used in older adults with stroke. The studies had moderate to relatively large sample sizes. Most of the tools were used in a hospital context but varied by hospital site and patient population. ISAR is a quick and easy screening tool widely used in the ED and acute care units. Moreover, the TRST was able to predict readmission as well. The HOSPITAL score, was a valuable predictor of hospital readmission for older adults, while RRAT was primarily geared toward patients in a high-risk group. Lastly, the LACE index is designed to quantify the risk of early death or unplanned readmission.

Tool's validity and reliability are vital in developing and testing a screening tool [26]. Validity for all 5 screening tools was reported in the past and was similar in the low-to-moderate range.

The variables most often incorporated as predictors in the tools were patient-oriented, such as cognitive and memory problems (8 studies); both the need for help and difficulties in walking (8 studies); and length of stay and comorbid conditions (4 studies). A large proportion of the tools included organizational predictors such as an extended stay of hospital admission history (12 studies) and polypharmacy ( 8 studies). The tools identified are only part of the intervention for attempting to prevent readmission. These should be a part of a system evaluation that will include organizational and systemlevel change for detecting multiple risks [27].

The present study employed a systematic review methodology and case report to accommodate data gathering in the health and medical sciences that enabled the significance of its findings. Strong evidence for good practice should be analyzed and synthesized by relevant stakeholder experts. It brings about strong tool development with improved adherence to obtain the most significant benefit from healthcare services; moreover, the quality of the tool must ensure applicability and efficacy. This design includes the selected case reports reflecting tool quality for content and construct validity. Case reports with case comparisons across 4 cases shared common traits of hospital readmission for stroke in elderly patients as a predictive goal of this phase. Comparative case studies involve analyzing and synthesizing the similarities, differences, and patterns, which leads to hospital readmission among the elderly with stroke.

Predictivity of RRST quality is attained if the high-risk group is identified correctly and which factors are of 'causal attribution' or 'casual contribution'. Comparative case studies covering 2 or more cases produce more generalizable knowledge about causal questions and a need to understand and explain how features within the context influence program or policy initiatives' success. Based on our results, continually monitoring lipids' alterations in 4 stroke elderly patients with concomitant 3 diseases appear extremely important on hospital readmission in 28 days [28]. In addition, a high risk ADL score shows that daily living activity needs should be managed carefully by counseling, raising perceived risk in daily eating, proper exercise, permanently reducing stress and medication adherence. The findings from case studies showed excessively long hospital stay indexes; case reports ranged from 7 - 17 days. Biomarker risks such as dyslipidemia's, arrhythmias, and anemia are a warning sign of hospital readmission of elderly stroke patients, increasing the mortality rate in Thailand [29]. This is an emerging concern not widely recognized in previous studies. Progressive increases in cholesterol may also be considered a marker of reversal of critical illness and patient recovery. Still, increasing cholesterol may induce or worsen underlying atherosclerotic cardiovascular diseases, stroke, or myocardial infarctions.

\section{Conclusions}

The RRST development is a crucial quality step in ensuring the achievement of intended quality outcomes. It is increasingly recognized that design should use systematic reviews, and be reorganized, criticized, and synthesized by crucial expertise in academic health sectors and health care services. Moreover, the 4 case reports reflect practicability, efficacy, and reliability for validity of prediction. The development of the RRST was achieved through research procedures required by a regulatory protocol for clinical practice. These standard guidelines will contribute to preparing a well-designed tool for clinical practice.

This study has some limitations. Evidence provided was limited by the small number of studies targeting older persons' stroke readmission in the same hospital over 28 days. Also, studies did not 
consider patients' career/work circumstances as a limitation on multiple risk prevention measures at home. Little work has been done in severe cases, especially regarding medical adherence practice and health outcome, including the acceptability of non-pharmacological approaches or alternative medicines using traditional cures.

Given the need for readmission screening of the elderly, we suggest researchers and healthcare workers providing stroke care use the tools we have reviewed in hospital readmission risk screening to plan for more effective healthcare services for elderly patients with stroke.

\section{Acknowledgments}

The National Research Council of Thailand and Faculty of Medicine Ramathibodi Hospital/ Ramathibodi School of Nursing financial support for the research, and we thank all the staff and patients who made this study possible.

\section{References}

[1] VL Feigin, MH Forouzanfar, R Krishnamurthi, GA Mensah, M Connor, DA Bennett, AE Moran, RL Sacco, L Anderson, T Truelsen, M O'Donnell, N Venketasubramanian, S Barker-Collo, CMM Lawes, W Wang, Y Shinohara, E Witt, M Ezzati, M Naghavi and C Murray. Global and regional burden of stroke during 1990 - 2010: Findings from the Global Burden of Disease Study 2010. Lancet 2014; 383, 245-54.

[2] MI Khan, JI Khan, SI Ahmed and U Haq. The epidemiology of stroke in a developing country (Pakistan). J. Neurol. Stroke 2018; 8, 00275.

[3] C Franchi, A Nobili, D Mari, D Mari, M Tettamanti, CD Djade, L Pasina, F Salerno, S Corrao, A Marengoni, A Iorio, M Marcucci and PM Mannucci. Risk factors for hospital readmission of elderly patients. Eur. J. Intern. Med. 2013; 24, 45-51.

[4] MF Kilkenny, M Longworth, M Pollack, C Levi and DA Cadilhac. Factors associated with 28-day hospital readmission after stroke in Australia. Stroke 2013; 44, 2260-8.

[5] JP Bettger, B Lupo, K Nichols, K Smith, E Windes and T Darden-Fluker. Hospital readmission among stroke patients who received post-hospital care: A systematic review. Int. J. Phys. Med. Rehabil. 2013; 1, 137.

[6] ML Barnett, J Hsu and JM McWilliams. Patient characteristics and differences in hospital readmission rates. JAMA Intern. Med. 2015; 175, 1803-12.

[7] D Moher, A Liberati, J Tetzlaff, and D Altman. Preferred Reporting items for systematic reviews and meta-analyess: The PRISMA statement. PLoS Med. 2009; 6, e1000097.

[8] R Farzanegan, B Farzanegan, M Alehashem, M Zangi, SRN Kalhori, K Sheikhy, H Emami and MB Shadmehr. Item selection and content validity of the risk factors of post-intubation tracheal stenosis observation questionnaire for ICU-admitted patients. Tanaffos 2017; 16, 22-33.

[9] CE Graf, SV Giannelli, FR Herrmann, FP Sarasin, JP Michef, D Zekry and T Chevalley. Identification of older patients at risk of unplanned readmission after discharge from the emergency department - comparison of two screening tools. Swiss Med. Wkly 2012; 141, w13327.

[10] T Braes, P Moons, P Lipkens, W Sterckx, M Sabbe, J Flammaing, S Boonen and K Milisen. Screening for risk of unplanned readmission in older patients admitted to hospital: Predictive accuracy of three instruments. Aging Clin. Exp. Res. 2010; 22, 345-51.

[11] P Moons, KD Ridder, K Geyskens, M Sabbe, T Braes, J Flamaing and K Milisen. Screening for risk of readmission of patients aged 65 years and above after discharge from the emergency department: Predictive value of four instruments. Eur. J. Emerg. Med. 2007; 14, 315-23.

[12] JD Donze, MV Williams, EJ Robinson, E Zimlichman, D Aujesky, EE Vasilevskis, S Kripalani, JP Metlay, GS Fletcher, AD Auerbach and JL Schnipper. Internal validity of the HOSPITAL score to predict 30-day potentially avoidable hospital readmissions. JAMA Inter. Med. 2016; 176, 496-502.

[13] T Fasolino and M Phillips. Utilizing risk readmission assessment tool for nonhospice palliative care consults in heart failure patients. J. Palliat. Med. 2016; 19, 1098-101.

[14] R Robinson. The HOSPITAL score as a predictor of 30 day readmission in a retrospective study at a university affiliated community hospital. PeerJ 2016; 4, e2441.

[15] S Boraiah, L Joo, IA Inneh, P Rathod, M Meftah, P Band, JA Bosco and R Iorio. Management of modifiable risk factors prior to primary hip and knee arthroplasty: A readmission risk assessment tool. J. Bone Joint Surg. Am. 2015; 97, 1921-8. 
[16] E Rosted, M Schultz, H Dynesen, M Dahl, M Sqrensen and S Sanders. The identification of seniors at risk screening tool is useful for predicting acute readmissions. Dan. Med. J. 2014; 61, A4828.

[17] K Singler, HJ Heppner, A Skutetzky, C Sieber, M Christ and U Thiem. Predictive validity of the identification of seniors at risk screening tool in a German emergency department setting. Gerontology 2014; 60, 413-9.

[18] JS Lee, G Schwindt, M Langevin, R Moghaghab, SMH Alibhai, A Kiss and G Naglie. Validation of the triage risk stratification tool to identify older persons at risk for hospital admission and returning to the emergency department. J. Am. Geriatr. Soc. 2008; 56, 2112-7.

[19] SW Meldon, LC Mion, RM Palmer, BL Drew, JT Connor, LJ Lewicki, DM Bass and CL Emerman. A brief risk-stratification tool to predict repeat emergency department visits and hospitalizations in older patients discharged from the emergency department. Acad. Emerg. Med. 2003; 10, 224-32.

[20] M Simpson. A quality improvement plan to reduce 30 -day readmissions of heart failure patients. $J$. Nurs. Care Qual. 2014; 29, 280-6.

[21] J Edmans, L Bradshaw, JRF Gladman, M Franklin, V Berdunov, R Elliott and SP Conroy. The Identification of Seniors at Risk (ISAR) score to predict clinical outcomes and health service costs in older people discharged from UK acute medical units. Age Aging 2013; 42, 747-53.

[22] CV Walraven, IA Dhalla, C Bell, E Etchells, IG Stiell, K Zarnke, PC Austin and AJ Forster. Derivation and validation of an index to predict early death or unplanned readmission after discharge from hospital to the community. Can. Med. Assoc. J. 2010; 182, 551-7.

[23] N Burns and S Grove. The practice of nursing research: Conduct, critique and utilization. $4^{\text {th }}$ eds. W.B. Saunders, Philadelphia, Pennsylvania, 2001.

[24] DE Hinkle, W Wiersma and SG Jurs. Applied statistics for the behavioral sciences. $5^{\text {th }}$ eds. Houghton Mifflin, Boston, MA, 2003.

[25] A Kumar. Review of the step for development of quantitative research tools. J. Adv. Practices Nurs. $2015 ; 1,103$.

[26] JL Yao, J Fang, QQ Lou and RM Anderson. A systematic review of the identification of seniors at risk (ISAR) tool for the prediction of adverse outcomes in elderly patients seen in the emergency department. Int. J. Clin. Exp. Med. 2015; 8, 4778-86.

[27] JR Vest, DL Gamm, BA Oxford, MI Gonzalez and KM Slawson. Determinants of preventable readmissions in the United States: A systematic review. Implement. Sci. 2010; 5, 88.

[28] C Qin, H Minghan, Z Ziwen and L Yukun. Alteration of lipid profile and value of lipids in the prediction of the length of hospital stay in COVID-19 pneumonia patients. Food Sci. Nutr. 2020; 8, 6144-52.

[29] N Lorking, AD Wood, S Tiamkao, AB Clark, K Kongbunkiat, JH Bettencourt-Silva, K Sawanyawisuth, N Kasemsap, MA Mamas and PK Myint. Seasonality of stroke: Winter admissions and mortality excess: A Thailand National Stroke population database study. Clin. Neurol. Neurosurg. 2020; 199, 106261. 